\title{
Metrische Eigenschaften der Curven dritter Ordnung mit einem Doppelpunkt.
}

Von Gottlieb Stiner in Zürich.

I.

1. Es seien gegeben ein Kegelschnitt $\mathscr{R}$, eine Gerade $a$ und auf dem Kegelschnitt ein fester Punkt $D$. Zieht man durch $D$ irgend einen Strahl $d$, so wird dieser den Kegelschnitt $\Omega$ zum zweiten Mal in einem Punkt $K$ treffen und die Gerade $a$ in einem Punkt $A$ schneiden. Construiert man auf jedem Strahl $d$ denjenigen Punkt $P$, für welchen

$$
D A=K P
$$

oder, was damit gleichbedeutend ist

$$
D P=D A+D K
$$

so ist der Ort von $P$ eine Curve dritter Ordnung, welche in $D$ einen Doppelpunkt hat.

Um dies zu beweisen, zieht man durch $K$ die Parallele $k$ zu $a$, welche dem Kegelschnitt in einem zweiten Punkt $K_{1}$ begegnet. Der Punkt $P_{1}$, für welchen $D A_{1}=K_{1} P_{1}$, liegt dann mit $P$ auf einer Geraden $p$, welche $z u a$ parallel ist und welche von $k$ dieselbe Entfernung hat wie $a$ von $D$. Dreht sich demnach $d$ um den Scheitel $D$, so beschreiben die Geraden $k$ und $p 2$ congruente gleichstimmige Parallelenbüschel. Die Strahlenpaare $d d_{1}$ bilden eine Involution, welche zu dem Büschel der Linien $k$ perspectivisch, somit zu dem Büschel der Linien $p$ projectivisch ist. Der definierte Ort von $P$ entsteht folglich als Erzeugniss der projectivischen $\mathrm{Zu}$ ordnung der Strahlenpaare einer Involution zu den Strahlen eines Büschels; er ist eine Curve dritter Ordnung $C_{3}$, für welche $D$ ein Doppelpunkt und die gemeinsame Richtung $A_{\infty}$ der Linien $p$ ein einfacher Punkt ist ${ }^{1}$ ).

Die symmetrische Gerade zu $a$ in Bezug auf $D$ sei $a^{*}$; diese begegne dem Kegelschnitt $\Re$ in den 2 Punkten $G$ und $H$. Dann sind die Strahlen $D G=g$ und $D H=h$ die Doppelpunktstangenten, weil die nach dem allgemeinen Verfahren auf diesen Strahlen lie-

1) E. Weyr: Theorie der mehrdeutigen geom. Elementargebilde, Leipzig 1869. 
genden Punkte der $C_{3}$ mit $D$ zusammenfallen. $D$ ist also ein eigentlicher Doppelpunkt, ein Rückkehrpunkt oder ein isolirter Doppelpunkt je nachdem $a^{*}$ den Kegelschnitt $\mathfrak{K}$ reell schneidet, beriihrt oder nicht reell schneidet.

2. Die Tangente in einem Punkt $P$ der $C_{3}$ ergibt sich durch folgende Betrachtung. Man nehme durch $D$ einen Strahl $d^{\prime}$ welcher mit $D P=d$ einen kleinen Winkel einschließt und construiere auf demselben den Punkt $P^{\prime}$ der $C_{3}$ nach der Bedingung $D A^{\prime}=K^{\prime} P^{\prime}$. Dann gibt es eine Hyperbel, welche $a$ und die Verbindungslinie $K K^{\prime}$ zu Asymptoten hat und durch die 3 Punkte $D, P, P^{\prime}$ geht. Dreht man jetzt $D P^{\prime}=d^{\prime}$ in der Richtung nach $d$, so nähert sich die Linie $K K^{\prime}$ der Tangente $t$ in $K$ an den gegebenen Kegelschnitt und die gemeinsame Sehne $P P^{\prime}$ der Hyperbel und der $C_{3}$ nähert sich der Tangente in $P$ an $C_{3}$. Im Grenzfall, wo $d^{\prime}$ mit $d$ zusammenfällt, fällt $K K^{\prime}$ mit $t$ zusammen und die Hyperbel und die $C_{3}$ berühren sich im Punkte $P$. Man erkennt daraus für die Tangente in $P$ an $C_{3}$ die tolgende Construction: sie ist die Tangente in $P$ an diejenige Hyperbel, welche durch $P$ geht und die beiden Geraden $a$ und $t$ zu Asymptoten hat. Construiert man also zum Schnittpunkt von $a$ und $t$ den symmetrischen in Bezug auf den Schnittpunkt von $p$ und $t$, so ist dieser ein Punkt der gesuchten Tangente. Der letztere Punkt kaun auch definiert werden als der symmetrische in Bezug auf $K$ zum Schnittpunkte von $t$ und $a^{*}$.

$\mathrm{Zu}$ dieser Tangentenconstruction kann man auch durch die folgende Überlegung kommen: Haben $K, K^{\prime}, P, P^{\prime}$ dieselbe Bedeutung wie vorhin, so gibt es auch eine Hyperbel, welche durch $D, K$ und $K^{\prime}$ geht and die Linien $a^{*}$ und $P P^{\prime}$ zu Asymptoten hat. Im Grenzfall, wo $d$ und $d^{\prime}$ zusammenfallen, wird die Linie $K K^{\prime}$ zur Tangente $t$ in $K$ an den gegebenen Kegelschnitt und die Asymptote $P P^{\prime}$ wird zur Tangente in $P$ an $C_{3}$. Die Tangente in $P$ an $C_{3}$ ist also dadurch definiert als die durch $P$ gehende Asymptote derjenigen Hyperbel, welche $a^{*}$ zur einen Asymptote hat und welche in $K$ den gegebenen Kegelschnitt berührt. Weil aber der Berührungspunkt einer Hyperbeltangente in der Mitte' des durch die Asymptoten aut der Tangente abgeschnittenen Segmentes liegt, so findet man also einen zweiten Punkt der gesuchten Asymptote, wenn man zum Schnittpunkt von $t$ mit $a^{*}$ den symmetrischen Punkt construiert in Bezug auf den Berührungspunkt $K$ von $t$ mit $\Re$.

Das Princip der letzten Tangenteneonstruction kann auch verwendet werden zur Bestimmung der 3 Schnittpunkte einer beliebigen Geraden $g$ mit der Curve $C_{3}$ : Es gibt eine Hyperbel, welche $a^{*}$ und $g \mathrm{zu}$ Asymptoten hat und durch $D$ geht; diese schneidet den gegebenen Kegelschnitt außer in $D$ noch in 3 weitern Punkten $K_{1}, K_{2}, K_{3}$. Die Strahlen $D K_{1}, D K_{2}$ und $D K_{3}$ treffen $g$ in den 3 gesuchten Punkten.

Aus den allgemeinen Tangentenconstructionen folgt speciell: a) Die gegebene Gerade $a$ ist die Tangente des Punktes $A_{\infty}$, also 
eine Asymptote der $\mathrm{C}_{3} ;$ b) die Asymptoten des gegebenen Kegelschnittes sind die 2 andern Asymptoten der $C_{3} ; c$ ) sind $K_{1}$ und $K_{2}$ die Berührungspunkte der zu $a$ parallelen Tangenten von $\mathfrak{N}$. so sind die Tangenten in den zugehörigen Punkten $P_{1}$ und $P_{2}$ der $C_{3}$ ebenfalls zu a parallel.

3. An die gegebenen Tangentenconstructionen schliesst sich noch Folgendes. Die erste Construction gibt Veranlassung zu dem Satz:

Die Enveloppedersämmtlichen Hyperbeln, welehe eine feste Gerade $a$ zur einen Asymptote haben, durch einen gegebenen Punkt $D$ gehen und deren zweite Asymptote einen durch $D$ gehenden festen Kegelschnitt berührt, ist eine Curve dritter Ordnung, für welche $D$ ein Doppelpunkt und a eine Asymptote ist; der Berührungspunkt irgend einer Hyperbel des Systems mit der Enveloppe liegt auf der Geraden von $D$ nach dem Berührungspunkt der zweiten Asymptote mit dem Kegelschnitt.

Die zweite Construction führt zu dem Satz:

Construiert man die sämmtlichen Hyperbeln, welche eine feste Gerade $a^{*}$ zur einen $\Delta$ symptote haben, durch einen Punkt $D$ geben und auberdem einen durch $D$ gehenden Kegelschnitt in irgend einem Punkte $K$ berühren, so ist die Enveloppeder zweiten Asymptoten dieser Hyperbeln eine Curve dritter Ordnung mit einem Doppelpunkt in D. Der Berührungspunkt irgend einer dieser Asymptoten liegt auf der Linie von $D$ nach dem Berihrungspunkt $K$ der zugehörigen Hyperbel mit dem gegebenen Kegelschnitt.

Dieser Satz liefert ein bequemes Mittel, um eine Curve dritter Ordnung mit einem Doppelpunkt ats Tangenten zu construieren.

Beide Sätze lassen sich projectivisch verallgemeinern. Die Verallgemeinerungen lauten:

a) Es seien gegeben zwei Gerade $a$ und $q$, ein Punkt $D$ und ein durch $D$ gehender Kegelschnitt $\Re$. Ist $t$ eine variable Tangente des gegebenen Kegelschnittes, so ist die Enveloppe derjenigen Kegelschnitte, welche durch $D$ gehen, ferner $a$ im Schnittpunkt $a q$ und $t$ im Schnittpunkt $t q$ berühren, eine Curvedritter Ordnung mit einem Doppelpunkt in $D$. Der Berührungspunkt der Enveloppe mit einem Kegelschnitt des Systems liegt auf derGeraden von $D$ nach dem Berührungspunkt ron $t$ mit dem gegebenen Kegelschnitt.

b) Es seien gegeben zwei Gerade $a^{*}$ und $q$, ein Punkt $D$ und ein durch $D$ gehender Kegelschnitt. 
Construiertmandiesämmtlichen Kegelschnitte, welche durch $D$ gehen, ferner $a^{*}$ im Schnittpunkt $a^{*} q$ und den Kegelsehnitt in irgend einem Punkt $K$ berühren, so umbïllen die Tangenten dieser Kegelschnitte in den resp. zweiten Schnittpunkten mit $q$ eine Curve dritter Ordnung, welche in $D$ einen Doppelpunkt hat. Der Berührungspunkt einer solchen Tangente mit der Enveloppe liegt auf der Verbindungslinie von $D$ mit dem zugehörigen Punkt $K$ des gegebenen Kegelschnittes.

Beide Sätze führen durch specielle Wahl der gegebenen Elemente zu interessanten Resultaten. Die dualistischen Übertragungen dieser zwei Sätze liefern Constructionen für die Curven dritter Classe mit einer Doppeltangente. Durch Specialisierung dieser dualistischen Übertragungen ergeben sich folgende Constructionen für die Steiner'sche Hypocykloide:

a) Es sei gegeben eine feste Parabel mit dem Brennpunkt $A^{*}$; $A$ sei ein beliebig gewählter Punkt der Ebene und $Q$ der Mittelpunkt der Strecke $A A^{*}$. Ist nun $T$ ein variabler Punkt der Parabel, so ist die Enveloppe der sämmtlichen Parabeln, welche die Gerade $A Q$ in $A$ und die Gerade $T Q$ in $T$ berühren, eine Steiner'sche Hypocykloide. Der Berührungspunkt einer Parabel des Systems mit der Enveloppe liegt auf derjenigen Tangente dieser Parabel, welche parallel ist zur Tangente im zugehörigen Punkte $T$ an die feste Parabel.

b) Es sei gegeben eine feste Parabel mit dem Brennpunkt $A^{*}$; $Q$ sei ein willkürlich angenommener Punkt der Ebene. Construiert man die sämmtlichen Parabeln, welche die Gerade $A^{*} Q$ in $A^{*}$ und die gegebene Parabel in irgend einem Punkte $T$ berühren, so liegen die Berührungspunkte $P$ der zweiten Tangenten dieser Parabeln aus $Q$ auf einer Steiner'schen Hypocykloide. Die Tangente in einem Punkte $P$ der Curve ist parallel mit der Tangente im zugehörigen Punkte $T$ an die feste Parabel.

Beide Sätze erfordern nur die Anwendung des Brianchon'schen Satzes. Aus diesen Sätzen ergibt sich auch die Beziehung, welche besteht zwischen drei Tangenten der Steiner'schen Hypocykloide, die durch einen Punkt $Q$ gehen und den zugehörigen Berührungspunkten. Sie lautet: Je zwei dieser Tangenten mit ihren Berührungspunkten bestimmen eine Parabel; die dritte Tangente geht durch den Brennpunkt dieser Parabel und der zugehörige Berührungspunkt ist der symmetrische zu diesem Brennpunkt in Bezug auf $Q$.

4. Es bleibt noch zu zeigen, dass jede $C_{3}$ mit einem Doppelpunkt, bei welcher der Doppelpunkt und eine Asymptote im Endlichen liegen, nach der in \$. 1 angegebenen Weise dargestellt werden kann. $D$ sei der Doppelpunkt, $a$ die Asymptote; $d$ ein Strahl durch den Doppelpunkt, $A$ dessen Schnittpunkt mit der 
Asymptote und $P$ dessen letzter Schnittpunkt mit $C_{3}$. Construiert man auf jedem Strahl $a$ durch $D$ denjenigen Pankt $K$, für welchen $D A=K P$, so ist der Ort von $K$ ein Kegelschnitt, welcher durch $D$ geht. Zum Beweis zieht man durch $P$ die Parallele $p$ zu $a$, welche der $C_{3}$ außer in $P$ und $A_{\infty}$ in einem dritten Punkt $P_{1}$ begegnet. Construiert man zu $P_{1}$ den Punkt $K_{1}$, so dass $D A_{1}=K_{1} P_{1}$, so liegen $K$ und $K_{1}$ auf einer Linie $k$, welche zu parallel ist und von ihr denselben Abstand hat wie $D$ von $a$. Wenn sich also $P$ verändert, so beschreiben $p$ und $k$ zwei congruente Parallelenbüschel. Nun bilden die Strahlenpaare $D P=d$ and $D P_{1}=d_{1}$ eine Involution, welche projectivisch ist zum Büschel der Linien $p^{1}$ ), also auch projectivisch zum Büschel der Linien $k$. Der Ort von $K$ entsteht also als Erzeugniss der ein-zweidentigen Zuordnung der Linien $k$ und $d$, ist demnach eine $C_{3}$. Fällt aber $p$ mit $a$ zusammen, so fällt der eine Strahl des zugehörigen Paares $d d_{1}$ zusammen mit der Parallelen durch $D$ zu a, mit welcher Geraden sich dann auch das entsprechende $k$ deckt. Diese Gerade sondert sich demnach als Bestandtheil der $C_{3}$ ab und es bleibt als eigentlicher Ort von $K$ ein Kegelschnitt, welcher durch $D$ geht.

Ist demnach eine $C_{3}$ gegeben durch den Doppelpunkt $D$, eine Asymptote $a$ und vier Punkte $P_{1} \ldots P_{4}$, so wird man zur weiteren Construction der Curve so verfahren: Man bestimmit auf den vier Strahlen $D P_{i}$ die Punkte $K_{i}$, für welche $D A_{i}=K_{i} P_{i}$, dann definieren diese vier Punkte $K_{i}$ mit $D$ einen Kegelschnitt. Construiert man jetzt auf jedem Strahl durch $D$ zum zweiten Schnittpunkt $K$ mit dem Kegelschnitt den Punkt $P$, für welchen $D A=K P$, so beschreibt $P$ die definierte $C_{3}$. Der construierte Kegelschnitt hat die zwei weiteren Asymptoten der $C_{3}$ zu seinen Asymptoten. Ist also speciell die Curve circular, so ist der Kegelschnitt ein Kreis und der Mittelpunkt des Kreises der ausgezeichnete Brennpunkt der Curve.

Sind von $C_{3}$ statt zweier Punkte $P_{i}$ ein Punkt und seine Tangente gegeben, so tritt eine geringe Modification der Construction des Kegelschnittes ein, welche sich aus $\$$. 2 ergibt.

Wenn die $C_{3}$ speciell gegeben ist durch den Doppelpunkt und die drei Asymptoten $a a^{\prime} a^{\prime \prime}$, so kann man als Kegelschnitt eine der drei Hyperbeln nehmen, welche zwei der gegebenen Geraden zu Asymptoten hat und durch $D$ geht. Man nehme z. B. die durch $D$ gehende Hyperbel, für welche $a^{\prime}$ und $a^{\prime \prime}$ die Asymptoten sind. $d$ sei ein beliebiger Strahl durch $D, A A^{\prime} A^{\prime \prime}$ seien seine Schnittpunkte mit $a a^{\prime} a^{\prime \prime}$. Dann erhält man auf $d$ den Punkt $K$, wenn man auf $d$ von $A^{\prime}$ aus eine Strecke abträgt, welche gleich und gleich gerichtet ist mit $D A^{\prime \prime}$, also $D K=D A^{\prime}+D A^{\prime \prime}$. Nun erhält man den auf $d$ liegenden Punkt $P$ der $C_{3}$ nach der Be-

1) Man vergl. E. Weyr a. a. O. 
ziehung $D A=K P$. Durch zweimaliges Abtragen von Strecken bekommt man also auf jedem durch $D$ gehenden Strahl den Punkt $P$. Nun ist

$$
\begin{aligned}
D P & =D K+K P \\
& =D K+D A \\
& =D A+D A^{\prime}+D A^{\prime \prime}
\end{aligned}
$$

Daraus ergibt sich der Satz: Ist $d$ ein beliebiger Strahl durch den Doppelpunkt $D$ einer $C_{3}, P$ sein Schnittpunkt mit der Curve, $A A^{\prime} A^{\prime \prime}$ seine Schnittpunkte mit den drei Asymptoten der Curve, so ist immer $\left.D P=D A+D A^{\prime}+D A^{\prime \prime} .{ }^{1}\right)$

Mit Hilfe dieser Construction von $P$ ergibt sich speciell noch die Bestimmung der Schnittpunkte der Asymptoten mit der Curve. Der Schnittpunkt ron $a$ mit $C_{3}$ ist der Schnittpunkt von $a$ mit der Tangente in $D$ an die oben betrachtete durch $D$ gehende Hyperbel, welche $a^{\prime}$ und $a^{\prime \prime}$ zu Asymptoten hat; entsprechend ergeben sich die Schnittpunkte mit $a^{\prime}$ und $a^{\prime \prime}$. Aus dieser Construction folgt: Liegt insbesondere $D$ auf einer Schwerlinie des durch die Asymptoten gebildeten Dreiecks, so ist die Seite des Dreiecks, welche durch diese Schwerlinie halbiert wird, eine W endeas ymptote der Curve. Fällt $D$ in den Schwerpunkt des Dreiecks, so sind die drei Asymptoten Wendeasymptoten.

Der Satz $D P=D A+D A^{\prime}+D A^{\prime \prime}$ lässt sich auch für den Fall beweisen, wo von den drei Asymptoten zwei, z. B. $a^{\prime}$ und $a^{\prime \prime}$ imaginär sind. Der Beweis kann so geliefert werden: $a_{i}^{\prime}$ und $a_{i}^{\prime \prime}$ sind conjugiert imaginär, also darstellbar als Doppelstrahlen einer elliptischen Involution $I$. Wir können dann die durch $D a a_{i}^{\prime}$ und $a_{i}^{\prime \prime}$ definierte $C_{3}$ construieren unter Benützung der Ellipse, welche durch $D$ geht und $I$ zu ihrer Durchmesserinvolution hat. Ein Strahl $d$ trifft diese Ellipse in einem Punkt $K$ und wir finden den zugehörigen Punkt $P$ nach der Beziehung $D P=D A+D K$. Nun ist aber $D K=2 D M$, wenn $M$ der Mittelpunkt der Involution harmonischer Pole von $d$ in Bezug auf die Ellipse ist. Man erhält $M$, wenn man den Mittelpunkt der Punktinvolution construiert, in welcher $d$ von der gegebenen Strahleninvolution $I$ geschnitten wird. Sind also $A_{i}^{\prime}$ und $A_{i}^{\prime \prime}$ die Doppelpunkte dieser Punktinvolution, d. h. die Schnittpunkte von $d$ mit $a_{i}^{\prime}$ und $a_{i}^{\prime \prime}$, so ist auch $D A_{i}^{\prime}+D A_{i}^{\prime \prime}=2 D M$; somit ist

$$
\begin{aligned}
& D K=D A_{i}^{\prime}+D A_{i}^{\prime \prime}, \text { oder } \\
& D P=D A+D A_{i}^{\prime}+D A_{i}^{\prime \prime} .
\end{aligned}
$$

1) Auf die weitgehenden Verallgemeinerungen dieses Satzes wird der Verfasser bei anderer Gelegenheit zu sprechen kommen. 
II.

5. Es seien wieder gegeben wie in $\& 1$ ein Kegelschnitt, eine Gerade $a$ und auf dem Kegelschnitt ein Punkt $D$. Construirt man auf jedem Strahl $d$ durch $D$ einen Punkt $P$ so dass $D K=P A$, so ist der Ort von $P$ ebenfalls eine Curve dritter Ordnung, welche in $D$ einen Doppelpunkt hat. Der Beweis hiefür kann auf dieselbe Art geliefert werden wie in $\$ 1$. Zwischen den zusammengehörigen Strahlen $k$ und $p$ besteht hier die Beziehung, dass die Mittellinie zwischen $k$ und $p$ immer auch die Mittellinie ist zwischen $a$ and $D$. Je zwei zusammengehörige Strahlen $k$ und $p$ liegen also symmetrisch in Bezug auf diese Mittellinie $m$. Man erkennt hieraus, dass der Ort von $P$ entsteht als Erzeugniss der projectivischen Zuordnung der Strahlen des Büschels vom Scheitel $A_{\infty}$ zu den Strahlenpaaren einer Involution vom Scheitel $D . P$ kann auch definiert werden als der symmetrische Punkt zu $K$ in Bezug auf den Schnittpunkt von $d$ mit $m$.

Aus der Construction folgt speciell: a) $C_{3}$ geht durch die Schnittpunkte von $m$ mit dem gegebenen Kegelschnitt. b) Sind $G$ und $H$ die Schnittpunkte von a mit dem gegebenen Kegelschnitt, so sind $D G=g$ und $D H=h$ die beiden Doppelpunktstangenten. $D$ ist demnach ein Knotenpunkt, ein Rückkehrpunkt oder ein isolirter Doppelpunkt, je nachdem $a$ den gegebenen Kegelschnitt reell schneidet, berührt oder nicht reell schneidet.

6. Um in $P$ die Tangente der $C_{3}$ zu construieren, nimmt man wieder einen Strahl $d$ ', welcher mit $d$ einen kleinen Winkel einschlief $3 t$ und construiert auf diesem den Punkt $P^{\prime}$, so dass $D K^{\prime}=P^{\prime} A^{\prime}$. Nun gibt es eine Hyperbel, welche durch die 4 Punkte $K K^{\prime} P P^{\prime}$ geht, für welche $a$ die eine Asymptote und $D$ ein Punkt der zweiten Asymptote ist. Dreht man den Strahl $d^{\prime}$ in der Richtung nach $d$, so bewegt sich $K^{\prime}$ auf dem Kegelschnitt in der Richtung nach $K$ und $P^{\prime}$ bewegt sich auf der $C_{3}$ in der Richtung nach $P$. Ist $d^{\prime}$ mit $d$ unendlich benachbart, so berührt die definierte Hyperbel in $K$ den gegebenen Kegelschnitt und in $P$ die $C_{3}$. Es ergibt sich also für die Tangente in $P$ an $C_{3}$ die folgende Construction: Sie ist die Tangente in $P$ an diejenige Hyperbel, welche a zur einen Asymptote hat, in $K$ den gegebenen Kegelschnitt berïhrt und durch $P$ geht. Nach einem bekannten Satz über die Hyperbel findet man einen zweiten Punkt der gesuchten Tangente, wenn man in Bezug auf $A$ den symmetrischen Punkt nimmt zum Schnittpunkt von $a$ mit der Tangente in $K$ an den gegebenen Kegelschnitt.

$\mathrm{Zu}$ demselben Punkt der gesucliten Tangente kann man auch auf folgende Weise kommen: Es gibt eine Hyperbel, welche die Linien $K K^{\prime}$ und $P P^{\prime}$ zu Asymptoten hat und durch die 3 Punkte $D A A^{\prime}$ geht. Wenn $d^{\prime}$ unendlich nahe zu $d$ rückt, so wird $K K^{\prime}$ zur Tangente $t$ in $K$ an den gegebenen Kegelschnitt, $P P^{\prime}$ zur Tangente in $P$ an $C_{3}$ und $A A^{\prime}=a$ zur Tangente in $A$ an die Hyperbel. Die gesuchte Tangente $\operatorname{der} C_{3}$ in $P$ ist somit definiert als 
die zweite Asymptote derjenigen Hyperbel, welche $t$ zur einen Asymptote hat, die Linie $a$ in $A$ beriihrt und durch $D$ geht. Man findet also einen zweiten Punkt der gesuchten Asymptote, wenn man in Bezug auf $A$ den symmetrischen nimmt zum Schnittpunkt von $a$ und $t$. Aus dieser Tangentenconstruction folgt noch: Die Linie $a$ ist die Tangente des Punktes $A_{\infty}$, also eine Asymptote. Die beiden anderen Asymptoten sind die symmetrischen Geraden in Bezug auf $D \mathrm{zu}$ den Asymptoten des gegebenen Kegelschnittes.

7. Analog den Sätzen von §. 3 ergibt sich hier:

a) Die Enveloppe der Hyperbeln, deren eine Asymptote eine feste Gerade $a$ ist, deren zweite Asymptote durch einen festen Punkt $D$ geht und welche überdies einen durch $D$ gehenden Kegelschnitt berühren ist eine $C_{3}$, welche $a$ zur einen Asymptote und $D$ zu einem Doppelpunkt hat. Der Berührungspunkt irgend einer Hyperbel des Systems mit der Enveloppe liegt auf der Linie von $D$ nach dem Berührungspunkt der Hyperbel mit dem festen Kegelschnitt.

b) Ist $D$ ein fester Punkt, $a$ eine feste Gerade, $A$ ein variabler Punkt derselben und $K$ der zweite Schnittpunkt von $D A$ mit einem dureh $D$ gehenden festen Kegelschnitt und construiert man für jede Lage von $A$ die Hyperbel, welche durch $D$ geht, $a$ in $A$ berüht und die Tangente in $K$ an den festen Kegelschnitt zur einen Asymptote hat, so ist die Enveloppe der zweiten Asymptote eine $C_{3}$, für welche $a$ eine Asymptote und $D$ der Doppelpunkt ist. Der Berührungspunkt einer solchen zweiten Asymptote liegt mit $D, A$ and $K$ in einer Geraden.

Auch hier können, wie in §. 3, die projectivischen Verallgemeinerungen und für diese die dualistischen Uebertragungen gebildet werden. Durch Specialisierung der Letzteren ergeben sich 2 weitere Constructionen der Steiner'schen Hypocykloide:

a) $A$ sei der Brennpunkt einer Parabel und $Q$ ein ganz beliebiger Punkt der Ebene. Construiert man die Hyperbeln, welche die Linie $A Q$ in $A$ und die Parabel in irgend einem Punkte $T$ berühren und deren eine Asymptote durch $Q$ geht, so ist die Enveloppe derselben eine Steiner'sche Hypocykloide. Die Berührungsstelle irgend einer Hyperbel des Systems mit der Enveloppe liegt auf derjenigen Tangente der Hyperbel, welche parallel ist zur Tangente in $T$ an die gegebene Parabel.

Es ist dabei nur noch zu bemerken, dass aus obigen Bedingungen für die Hyperbel die durch $Q$ gehende Asymptote gefunden werden kann als die Parallele zum vierten harmonischen Strahl zu $T A$ in Bezug auf $T Q$ und die Tangente in $T$ an die Parabel.

b) $A$ sei der Brennpunkt einer Parabel, $k$ sei eine beliebige Tangente derselben mit dem Berührungspunkt $T$, a die Parallele 
zu $k$ durch $A$, endlich $Q$ ein ganz willkürlicher Punkt der Ebene. Construiert man für jede Lage von $k$ diejenige Parabel, welche $Q T$ in $T$ und $a$ in $A$ berührt, so beschreibt der Berührungspunkt der zweiten Tangente aus $Q$ an diese Parabel eine Steiner'sche Hypocykloide. Die Tangente eines solchen Punktes ist immer parallel zur zugehörigen Lage von $k$.

Einfache Constructionen der Hypocykloide ergeben sich auch aus den Ausgangsconstructionen in den $\$ \S .1$ und 5 .

8. In $\$ .5$ wurde gezeigt, dass die Doppelpunktstangenten $g$ und $h$ dér $C_{3}$ die Verbindungslinien von $D$ mit den Schnittpunkten $G$ und $H$ von $a$ mit dem gegebenen Kegelschnitt sind. Nun sind die Doppelpunktstangenten die Doppelstrahlen derjenigen Involution, welche gebildet wird durch die Verbindungslinien der Paare conjugierter Punkte der $C_{3}$ mit dem Doppelpunkt ${ }^{1}$ ). Ist also $X X_{1}$ ein beliebiges Paar der Involution harmonischer Pole von $a$ in Bezug auf den gegebenen Kegelschnitt, so gehen die Strahlen $D X$ und $D X_{1}$ nach einem Paar conjugierter Punkte der $C_{3}$. Die zweiten Schnittpunkte von $D X$ und $D X_{1}$ mit dem gegebenen Kegelschnitt seien $\mathfrak{F}_{\mathcal{B}}$ und $\mathfrak{P}_{1}$, die ihnen zugehörigen Punkte der $C_{3}$ seien $P$ und $P_{1}$, also $D \mathfrak{B}=P X$ und $D \mathfrak{B}_{1}=P_{1} X_{1}$. Die Verbindungslinie $\mathfrak{P} \mathfrak{P}_{1}$ geht durch den $\mathrm{Pol} P_{a}$ von $a$ in Bezug auf den gegebenen Kegelschnitt and die Tangenten in $\mathfrak{P}_{\mathrm{B}}$ und $\mathfrak{P}_{1}$ an den gegebenen Kegelschnitt schneiden sich in einem Punkte $\mathfrak{I}$ von $a$. Construiert man mit Hilfe dieser Tangenten nach $\$ 6$ die Tangenten $t$ und $t_{1}$ in $P$ und $P_{1}$ an $C_{3}$ so findet man einen Punkt von $t$, wenn man zu $\mathfrak{I}$ den symmetrischen Punkt nimmt in Bezug: auf $X$ und man findet einen Punkt von $t_{1}$, wenn man zu $\mathfrak{T}$ den symmetrischen Punkt nimmt in Bezug auf $X_{1}$. Aus dieser Construction folgt, dass das Segment von $a$ zwischen diesen 2 symmetrischen Punkten doppelt so grol ist wie das Segment $X X_{1}$ und man hat damit den Satz: Das Segment, welches die Tangenten in 2 conjugierten Punkten einer $C_{3}$, bei welcher $D$ und $a$ im Endlichen liegen, auf einer Asymptote abschneiden, ist immer gleich gerichtet und oppeltsogrob wie das Segment, welches auf derselben Asymptote abgeschnitten wird durch die Verbindungslinien dieser conjugierten Punkte mit dem Doppelpunkt. ${ }^{2}$ )

Die Tangenten $t$ und $t_{1}$ schneiden sich in einem neuen Punkt $T$ der $C_{3}$, dem Tangentialpunkt von $P$ und $P_{1}$. Durch unsere Construction ist nun jedem Punkt $\mathfrak{T}$ der Linie $a$ ein bestimmter Punkt $T$ der $C_{3}$ zugeordnet und umgekehrt. Man kann zeigen, dass die $\mathrm{Zu}$ ordnung eine projectivische ist. Die Paare der Strahleninvolution $D P, D P_{1}$ sind projectivisch zugeordnet den Strahlen $D T^{3}$ ); letzteres

1) Man vergl. E. Weyr a. a. O.

2) Dass jede $C_{3}$, bei welcher $D$ und $a$ im Endlichen liegen, auf die Weise des §. 5 dargestellt werden kann, wird auf analoge Art bewiesen wie in $\S .4$.

3) Man vergl. E. Weyr a. a: 0 . 
Büschel ist also projectivisch zu dem Büschel der Linien $\mathfrak{P} \mathfrak{P}_{1}$ durch den Pol $P_{a}$. Dieses Büschel endlich ist projectivisch zur Reihe seiner Pole $\mathfrak{T}$ auf $a$. Daraus folgt also, dass die Reihe der Punkte $\mathfrak{T}$ projectivisch ist zum Büschel der Geraden $D T$. Die ein-zweideutige Beziehung, welchebesteht zwischen den Paaren conjugierter Punkte der $C_{3}$ und den zugehörigen Tangentialpunkten ist also damit eindeutig übertragen auf den gegebenen Kegelschnitt und die.Gerade $a$, in der Weise, dass der Kegelschnitt der Träger der zweideutigen Reihe und die Gerade $a$ der Träger der eindeutigen Reihe ist. Es ist noch zu bemerken, dass 2 Punkte $\mathfrak{I}$ und $\mathfrak{I}_{1}$ von $a$ die correspondierenden von 2 conjugierten Punkten $T$ und $T_{1}^{\prime}$ der $C_{3}$ sind, wenn sie conjugierte harmonische Pole sind in Bezug auf den gegebenen Kegelschnitt.

Die obige Übertragung führt zu folgender Betrachtung: Legt man durch den Punkt $T$ der $C_{3}$ einen veränderlichen Strahl, welcher der Curve in jeder Lage noch in 2 Punkten $P^{\prime}$ und $P^{\prime \prime}$ begegnet, so bilden die Strahlenpaare $D P^{\prime}, D P^{\prime \prime}$ eine Involution, deren Doppelstrahlen die Linien $D P$ und $D P_{1}$ sind. ${ }^{1}$ ) Daraus folgt, dass die Verbindungslinien der zugehörigen Punkte $\mathfrak{B}^{\prime}$ und $\mathfrak{P}^{\prime \prime}$ auf dem Kegelschnitt durch $\mathfrak{T}$ gehen müissen. Man kann zeigen, dass die Zuordnung der Strahlen $\mathfrak{P}^{\prime} \mathfrak{P}^{\prime \prime}$ zu den Strahlen $P^{\prime} P^{\prime \prime}$ eine projectivische ist. Das Büschel der Linien $\mathfrak{P}^{\prime} \mathfrak{P}^{\prime \prime}$ ist nämlich perspectivisch zu der Strahleninvolution $D \mathfrak{P}^{\prime}, D \mathfrak{P}^{\prime \prime}$ und diese Involution ist wiederum projectivisch zu dem Büschel der Linien $P^{\prime} P^{\prime \prime}$. Zi eht man also in der Ebene irgend eine Gerade, welche dem Kegelschnitt in 2 Punkten $\mathfrak{F}^{\prime}$ und $\mathfrak{P}^{\prime \prime}$ und der Geraden $a$ in einem Punkt $\mathfrak{I}$ begegnet, so liegen die Punkte $P^{\prime}$ und $P^{\prime \prime}$ in gerader Linie mit dem Punkt $T$ der $C_{3}$, welcher $\mathfrak{I}$ correspondiert.

9. Mit Hilfe der Resultate des vorhergehenden Paragraphen lässt sich eine vollständige Theorie der conjugierten Punkte der $C_{3}$ einfach ableiten aus den Involutionseigenschaften des gegebenen Kegelschnittes.

Einige Beispiele mögen dies zeigen:

1. Verbindet man zwei Punkte $\mathfrak{B}$ und $\mathfrak{P}_{1}$ des gegebenen Kegelschnittes, deren Verbindungslinie durch den Pol $P_{a}$ von $a$ geht, mit einem beliebigen Punkte $\mathfrak{T}$ von $a$, so treffen diese Verbindungslinien den Kegelschnitt zum zweitenmal in zwei neuen Punkten $\mathfrak{P}^{\prime}$ und $\mathfrak{P}_{1}^{\prime}$, deren Verbindungslinie durch $P_{a}$ geht. Daraus folgt: Verbindet man zwei conjugierte Punkte der $C_{3}$ mit einem beliebigen Curvenpunkt $T$, so sind die dritten Schnittpunkte dieser zwei Verbindungslinien wieder ein Paar conjugierter Punkte $P^{\prime}$ und $P_{1}^{\prime}$ der $C_{\hat{3}}$.

1) Man vergl. E. Weyx a. a. O. 
2. Verbindet man noch $\mathfrak{P} \mathfrak{P}_{1}^{\prime}$ und $\mathfrak{P}_{1} \mathfrak{P}^{\prime}$; so schneiden sich diese zwei Geraden in einem Punkt $\mathfrak{I}_{1}$ von $a$, welcher der conjugierte ist $z \mathrm{u} \mathfrak{I}$ in der Involution harmonischer Pole auf $a$ in Bezug auf den gegebenen Kegelschnitt. Daraus folgt: Verbindet man $P P_{1}^{\prime}$ and $P^{\prime} P_{1}$, so schneiden sich diese Linien in einem Punkt $T_{1} \operatorname{der} C_{3}$, welcher der conjugierte ist von $T$.

3. Sind $\mathfrak{B}$ und $\mathfrak{B}_{1}$ zwei Punkte des. Kegelschnittes, deren Tangenten sich in einem Punkt $\mathfrak{I}$ von $a$ schneiden, so ist der Schnittpunkt der Geraden $\mathfrak{P} \mathfrak{P}_{1}$ mit a der conjugierte Punkt zu $\mathfrak{I}$ in der Involution harmonischer Pole von $a$ in Bezug auf den Kegelschnitt. Daraus folgt: Die Verbindungslinie von zwei conjugierten Punkten $P P_{1}$ trifft die $C_{3}$ in einem neuen Punkt $T_{1}$,' welcher der conjugierte ist zum Tangentialpunkt $T$ von $P$ und $P_{1}$.

Die sechs Punkte $P P_{1} P^{\prime} P_{1}^{\prime} T T_{1}$ in Beispiel 2. sind die Ecken eines vollständigen Vierseits. Die Sätze von \$. 8 geben newe Gesichtspunkte für die Schliefungsprobleme überhaupt.

\section{III.}

Die in den $\$ \S .1$ und 5 entwickelten Constructionen der $C_{3}$ sollen noch verwendet werden zur Construction der beiden bekanntesten Curven dritter Ordnung mit einem Doppelpunkt, der Strophoide und der Cissoide. ${ }^{1}$ )

\section{Die Strophoide.}

10. Der gegebene Kegelschnitt sei ein Kreis vom Mittelpunkt $M ; D$ sei ein beliebiger Punkt des Kreises, $O$ der symmetrische Punkt zu $M$ in Bezug auf $D$ und endlich $a$ die Senkrechte in $O$ zum Durchmesser $O M$. Construiert man hier auf jedem Strahl durch $D$ einen Punkt $P$, so dass $D K=A P$, so ist der Ort von $P$ eine Strophoide. $a$ ist eine Wendeasymptote der Curve; die beiden andern Asymptoten sind die imaginären Asymptoten des Kreises vom Mittelpunkt $M$. Weil $M$ auf der Strophoide liegt, so sind also die Kreispunkte conjugierte Punkte der Curve. Letzteres hat zur Folge, dass die Doppelpunktstangenten $g$ und $h$ zu einander senkrecht stehen, was durch die allgemeine Construction derselben bestätigt wird. Verbindet man $P$ und $K$ mit $M$, so ist $\triangle A O D \cong \triangle P M K$, weil $O D=M K=r$, $A D=P K$ und $\Varangle A D O=\Varangle K D M=\Varangle P K M$. Aus der Congruenz folgt: $\Varangle P M K=90^{\circ}$. Ist $K^{\prime}$ der zu $K$ diametral gegenïberliegende Punkt im Kreis, so liegt zufolge dieser Eigenschaft $P^{\prime}$ ebenfalls auf MP. Man bekommt damit den Satz:

1) Man vergl. dazu hauptsächlich: M. G. de Long champs: Cours do Mathématiques speciales, IIe partie, $2^{\mathrm{e}}$ et $3^{\mathrm{e}}$ leçons mit den zugehörigen Übungen.'

Ferner: Baltzer: Analytische Geometrie, \$. 26. 
Projiciert man von irgend einem Punkt $D$ eines Kreises aus die Endpunkte jedes Durchnessers auf den dazu senkrechten Durchmesser, so ist der Ort dieser Projectionen eine Strophoide. Diese Construction kann auch so aufgefasst werden:

Bei einem veränderlichen rechtwinkligen Dreieck $P M K$, dessen eine Kathete $M K$ von constanter Länge ist, drehe sich der Scheitel des rechten Winkels um einen festen Punkt $M$ und die Hypotenuse drehe sich un einen festen Punkt $D$, welcher der Bedingung genügt, dass $M D=M K$, dann beschreibt die letzte Ecke $P$ eine Strophoide.

Man kann für diese Construction einen einfachen Mechanismus herstellen.

$B$ sei der Schnittpunkt von $M P$ mit $a$, dann ist $\Varangle B A P=\Varangle B P A$, weil beide gleich sind mit $\Varangle K P M$. Das Dreieck $A B P$ ist also ein gleichschenkliges. Man hat damit den Satz:

Verändert sich ein gleichschenkliges Dreieck so, dass der eine Schenkel $A B$ sich auf der festen Geraden $a$ bewegt, während der andere Schenkel $B P$ sich um einen festen Punkt $M$ und die Basis $A P$ sich um einen festen Punkt $D$ dreht, so ist der Ort der Ecke $P$ eine Strophoide.

Man kann diese Construction dadurch verallgemeinern, dass man an Stelle von $a$ irgend eine dazu parallele Gerade $p$ nimmt. Lässt man $p$ speciell mit der Tangente $d$ in $D$ an den gegebenen Kreis zusammenfallen, so folgt:

Bleiben von einem gleichschenkligen Dreieck $D C P$ die Ecke $D$ and die Richtung des einen Schenkels $D C$ fest, während der andere Schenkel $C P$ durch einen festen Punkt $M$ geht, welcher auf der Senkrechten in $D$ zu $C D$ liegt, so ist der Ort von $P$ eine Strophoide.

Ist also $C$ ein beliebiger Punkt von $d$, so bekommt man die zwei Punkte $P$ und $P^{t}$ der Curve, welche auf $C M$ liegen, so, dass man auf $C M$ von $C$ aus nach beiden Seiten die Länge $C D$ abträgt. Man kann dies auch so ausdrücken:

Die Endpunkte der durch $M$ gehenden Durchmesser sämmtlicher Kreise, welche durch $D$ gehen und ihre Mittelpunkte auf $d$ haben, liegen auf einer Strophoide.

Es ergibt sich noch, dass auf jedem Strahl durch $M$ die Entfernungen $M P$ und $P^{\prime} B$ einander gleich sind.

Nimmt man in obiger Construction zwei Kreise, deren Centra $C$ und $C_{1}$ symmetrisch zu $D$ liegen, so erhält man 4 Punkte der $C_{3} P P^{\prime} P_{1} P_{1}^{\prime}$, welche paarweise symmetrisch zu $O M$ liegen; die Verbindungslinien $P P_{1}$ und $P^{\prime} P_{1}^{\prime}$ sind also parallel zu $a$ und liegen symmetrisch in Bezug auf $D$. Man kann zeigen, dass die 4 Geraden $P P^{\prime}, P_{1} P_{1}^{\prime}, P P_{1}$ und $P^{\prime} P_{1}^{\prime}$ Tangenten eines Kreises 
vom Centrum $D$ sind. Es ist nämlich $\Varangle C D P=\Varangle P^{\prime} P D=$ $=\Varangle D P P_{1}$; also ist $D P$ die Halbierungslinie des $\Varangle P^{\prime} P P_{1}$, womit der Satz bewiesen ist. Daraus folgt der Satz:

Schneidet man die Tangenten aus $M$ an irgend einen Kreis vom Centrum $D$ mit den zwei zu $M D$ senkrechten Tangenten desselben Kreises, so ist der Ort dieser Schnittpunkte eine Strophoide.

Eine weitere Eigenschaft der Strophoide ergibt sich aus der Betrachtung der 2 letzten Seiten $P P_{1}^{\prime}$ und $P^{\prime} P_{1}$ des Vierecks $P P_{1} P^{\prime} P_{1}^{\prime} . \quad m$ sei die Senkrechte in $M$ zu $M D, Q$ und $Q_{1}$ die Schnittpunkte derselben mit $P P_{1}^{\prime}$ und $P_{1} P^{\prime}$, ferner $F$ der symmetrische Punkt zu $D$ in Bezug auf $M$. Dann ist $D Q \perp P_{1} P^{\prime}$ und $D Q_{1} \perp P P_{1}^{\prime}$, weil $Q$ und $Q_{1}$ die Pole sind von $P_{1} P^{\prime}$ und $P P_{1}^{\prime}$ in Bezug auf den Kreis, welcher die Linien $P P^{\prime}, P_{1} P_{1}^{\prime}, P P_{1}$ and $P^{\prime} P_{1}^{\prime}$ beriihrt. Weiter ist nun $\left.P P_{1}^{\prime}\right\lrcorner F Q$ und $P^{\prime} P_{1} \perp F Q_{1}, \mathrm{~d}$.h. die Linien $P P_{1}^{\prime}$ und $P_{1} P^{\prime}$ sind Tangenten derjenigen Parabel, für welche $F$ der Brennpunkt und $M$ der Scheitel ist.

Die Geraden $D Q$ and $P_{1} P^{r}$ schneiden sich in einem Punkt $P^{*}$ und die Geraden $D Q_{1}$ und $P P_{1}^{\prime}$ schneiden sich in einem Punkt $P_{1}^{*}$ Die Verbindungslinie $P^{*} P_{1}^{*}$ ist die Polare von $M$ in Bezug auf den Kreis, welcher durch $P^{*} P_{1}^{*}$ und $D$ geht, d. h. die Linien $M P^{*}$ und $M P_{1}^{*}$ sind die Tangenten aus $M$ an diesen Kreis. Daraus folgt aber, dass $P^{*}$ und $P_{1}^{*}$ ebenfalls auf der Strophoide liegen, denn sind $C^{*}$ und $C_{1}^{*}$ die Schnittpunkte von $M P^{*}$ and $M P_{1}^{*}$ mit $d$, so sind die Längen $C^{*} D$ und $C^{*} P^{*}$, sowie $C_{1}^{*} D$ und $C_{1}^{*} P_{1}^{*}$ einander gleich. Nach einem früheren Satz ist damit bewiesen, dass $P^{*}$ und $P_{1}^{*}$ der Strophoide angehören.

Die Construction von $P^{*}$ und $P_{1}^{*}$ führt zu den Sätzen:

Die Berührungspunkte der Tangenten aus $M$ an die Kreise, welche durch $D$ gehen und die Mittelpunkte auf $M D$ haben, liegen auf einer Strophoide.

Der Ort der Höhenfufpnnkte der gleichschenkligen Dreiecke $Q D Q_{1}$, welche ibre Spitze in $D$ und ihre Basis anf der Geraden $m$ haben, ist eine Strophoide.

Die Strophoide ist die Fußpunktscurve der Parabel rom Brennpunkt $F$ und vom Scheitel $M$ für den Punkt $D$, wobei $D$ der Schnittpunkt der Axe mit der Directrix der Parabel ist.

\section{Die Cissoide. $\left.{ }^{1}\right)$}

11. Der gegebene Kegelsehnitt sei ein Kreis vom Mittelpunkt $M$ und dem Radius $r ; D$ sei ein beliebiger Punkt des Kreises, $F$

1) Man vergl. Kl ügel: Math. Wörterbuch, I. Theil, Seite 434. 
der diametral gegenüberliegende und $O$ der symmetrische zu $F$ in Bezug auf $D$, also $D O=2 r$, endlich $a$ die Senkrechte in $O$ zu $O M$. Construiert man nun zu jedem Punkt $K$ des Kreises einen Punkt $P$, so dass $D K=A P$, so ist der Ort von $P$ eine Cissoide. $D$ ist ein Rückkehrpunkt der Curve, $D M$ die zugehörige Rückkehrtangente, $a$ eine Asymptote und zwar speciell eine Wendeasymptote; die beiden andern Asymptoten sind die imaginären Asymptoten des Kreises. Ist $K^{\prime}$ der diametral gegenüber liegende Punkt zu $K$ im gegebenen Kreis, so ist $\triangle A O D \cong \triangle P K^{\prime} K$, weil $A D=P K, O D=K K^{\prime}=2 r$ und $\Varangle A D O=\Varangle M D K=$ $=\Varangle P K K^{\prime}$. Aus der Congruenz folgt: $\Varangle P K^{\prime} K=90^{\circ}$, d. h. $P K^{\prime}$ ist die Tangente in $K^{\prime}$ an den gegebenen Kreis. Aus der Congruenz folgt also der Satz:

Projiciert man aus irgend einem Punkte $D$ eines Kreises jeden Peripheriepunkt $K$ auf die Tangente des diametral gegeniuberliegenden Punktes $K^{\prime}$, so ist der Ort dieser Projectionen eine Cissoide.

Diese Construction kann auch so ausgedrückt werden:

Bewegt sich ein veränderliches rechtwinkliges Dreieck $P K^{\prime} K$ so, dass die eine Kathete $K K^{\prime}$ von constanter Länge sich um ihren Mittelpunkt $M$ dreht, während die Hypotenuse $K P$ sich um einen festen Punkt $D$ dreht, dessen Entfernung von $M=\frac{1}{2} K K^{\prime}$, so ist der Ort der Ecke $P$ eine Cissoide.

Diese Bewegung kann durch einen einfachen Mechanismus veranschaulicht werden. Die obige Construction kann man auch so auffassen:

Der Scheitel des rechten Winkels eines veränderlichen rechtwinkligen Dreiecks $P D K$ drehe sich um einen festen Punkt $D$, der Endpunkt $K$ der einen Kathete bewege sich auf irgend einem durch $D$ gehenden Kreis und die Hypotenuse sei die Tangente in $K$ an diesen Kreis, dann beschreibt die letzte Ecke $P$ eine Cissoide.

Man erkennt in den letzten Constructionen die Darstellung der Cissoide als Erzeugniss der projectivischen Zuordnung der Tangenten des festen Kreises zu den Strahlen des Büschels vom Scheitel $D$.

Ist $T$ der Schnittpunkt der Tangente $t^{\prime}$ in $K^{\prime}$ mit der Tangente $d$ in $D$ an den gegebenen Kreis, so ist das Dreieck $D T K^{\prime}$ gleichschenklig, also auch das Dreieck DTP. Es sind demnach die Strecken $T D, T K^{\prime}$ und $T P$ einander gleich. Man bekommt damit den Satz:

Sind $d$ eine feste und $t^{\prime}$ eine variable Tangente eines Kreises und nimmt man immer zum Berührungspunkt $K^{\prime}$ von $t^{\prime}$ mit dem $\mathrm{Kr}$ reis den symmetrischen Punkt $P$ in Bezug auf den Schnittpunkt von $t^{\prime}$ und $d$, so beschreibt $P$ eine Cissoide. 
Der Punkt $T$ ist das Centrum eines Kreises, welcher durch $P, D$ und $K^{\prime}$ geht und man bekommt den Satz:

$D$ sei ein Punkt eines festen Kreises, $d$ seine Tangente und $T$ ein variabler Punkt derselben; construiert man auf dem Kreis, welcher $T$ zum Centrum hat und durch $D$ geht den diametral gegenüberliegenden Punkt $P$ zum Schnittpunkt $K^{\prime}$ mit dem festen Kreis, so ist der Ort von $P$ eine Cissoide.

Man erkennt in dieser Construction die Entstehung der Cissoide aus der projectivischen Zuordnung der Elemente eines Kreisbüschels zu den Elementen eines Strahlenbüschels.

Der obige Kreis durch $D$ vom Centrum $T^{\prime}$ trifft die Linie $d$ in einem zweiten Punkt $E$. Die vier Punkte $D K^{\prime} E P$ bilden die Ecken eines Rechtecks; es geht also die Seite $K^{\prime} E$ durch den Punkt $F$, den zweiten Endpunkt des Durchmessers $D M$ im festen Kreis. Verändert man $T$, so bewegt sich $E$ auf $d, K^{\prime}$ auf dem festen Kreis und die Seite $K^{\prime} E$ dreht sich um den festen Punkt $F$. Es folgt daraus:

Bewegt sich ein veränderliches Rechteck so, dass die Ecke $D$ und die Diagonale $D E=d$ fest bleiben, während die Seite $E K^{\prime}$ sich um den festen Punkt $F^{\prime}$ dreht, so beschreibt die letzte Ecke $P$ dieses Rechtecks eine Cissoide.

Die letzte Construction kann noch einfacher ausgedrückt werden. Die Linie EP umhüllt bei der Bewegung des Rechtecks eine Parabel, für welche $F$ der Brennpunkt und $D$ der Scheitel ist. Die Cissoide erscheint demnach als Fußpunktscurve einer Parabel für den Scheitel derselben als Pol. Die Wendeasymptote der Cissoide ist dabei identisch mit der Directrix der Parabel.

$m$ sei die Parallele durch $M$ zu $a, M^{\prime}$ ihr Schnittpunkt mit der Tangente $t^{\prime}$ in $K^{\prime} ; n$ sei die Parallele zu $a$ durch den Mittelpunkt der Strecke $O D, N^{\prime}$ ihr Schnittpunkt mit $t^{\prime}$ und endlich $N$ der Schnittpunkt von $n$ mit der Normale in $P$ zu $t^{\prime}$. Dann ist $\triangle N P N^{\prime} \cong \triangle M K^{\prime} M^{\prime}$, denn die Strecken $P N^{\prime}$ und $K^{\prime} M^{\prime}$ haben dieselbe Länge, die Winkel bei $P$ und $K^{\prime}$ sind rechte und die Winkel bei $N$ und $M$ sind einander gleich, weil ihre Schenkel resp. parallel laufen. Aus der Congruenz folgt: $N P=K^{\prime} M=r$, also constant für alle Punkte der Curve.

Hat also bei einem rechten Winkel $N P K^{\prime}$ der eine Schenkel $N P$ die constante Länge $r$ und bewegt sich dieser rechte Winkel so, dass der Punkt $N$ die Gerade $n$ durchläuft, während der andere Schenkel $P K^{\prime}=t^{\prime}$ den festen Kreis berührt, jedoch immer so, dass $N$ und der Mittelpunkt $M$ des Kreises auf verschiedenen Seiten von $t^{\prime}$ liegen, so beschreibt der Scheitel des rechten Winkels eine Cissoide.

Zieht man noch durch $M$ die Parallele zu $t^{\prime}$ und $t$, welche $P N$ in dem Punkte $S$ begegnet, so ist $N S=2 r$ und man hat den Satz: 
Hat bei einem rechten Winkel NSM der eine Schenkel $N S$ die constante Länge $2 r$ und bewegt sich der Punkt $N$ auf der Linie $n$, während der andere Schenkel $S M$ durch den festen Punkt $M$ geht, so beschreibt der Mittelpunkt der Strecke NS eine Cissoide. ${ }^{1}$ )

Es kann hier noch eine einfache Construction der Cissoide mit Hilfe einer constanten Zirkelöffnung erwähnt werden. Nach dem Vorigen ist $N P=r=M K$, ferner ist $D K=A P$ und $\Varangle D K M=\Varangle A P N$. Somit ist $\triangle D K M \cong \triangle A P N$, also. $\Varangle K D M=\Varangle P A N$, d. h. $A N$ steht rechtwinklig auf $a$ und seine Länge ist gleich $r$.

Verschiebt man also ein veränderliches gleichschenkliges Dreieck $A N P$, dessen Schenkel $A N$ und $N P$ die constante Länge $r$ haben, so, dass $A$ und $N$ sich auf $a$ und $n$ bewegen, während die Basis $A P$ durch $D$ geht, so beschreibt $P$ eine Cissoide.

Eine ähnliche Construction besteht mit Hilfe eines rechtwinkligen Dreiecks. Die Verlängerung von $A N$ geht nämlich durch den früher benutzten Punkt $E$. Man hat also ein rechtwinkliges Dreieck $A P E$, bei welchem die Hypotenuse die constante Länge $2 r$ hat, während die eine Kathete $A P$ durch den festen Punkt $D$ geht. Es folgt der Satz:

Verschiebt man ein veränderliches rechtwinkliges Dreieck $\triangle P E$, dessen Hypotenuse $A E$ die constante Länge $2 r$ hat, so, dass $A$ und $E$ sich auf $a$ und $d$ bewegen, während die Kathete $A P$ durch den festen Punkt $D$ geht, so beschreibt. der Scheitel des rechten Winkels eine Cissoide.

Analoge Constructionen existieren für alle circularen $C_{3}$ mit einem Doppelpunkt.

Zum Schluss sei dem Verfasser noch die Bemerkung erlaubt, dass die vorliegende Arbeit über die $C_{3}$ als ein Beitrag zur Theorie einer durch einen Punkt $D$ und eine Gerade $a$ vermittelten Transformation zu betrachten ist. Diese Transformation wird auch für gewisse Curven höherer Ordnung ein nicht unwesentliches Untersuchungsmittel liefern. ${ }^{2}$ )

1) Es ist die Newton'sche Construction der Cissoide.

2) Man vergl. auch Ch. Beyel: "Ưtber Curven IV. Ordnung mit einem doppelten Berührungsknoten und einem Doppelpunkt", Art. 7, Vierteljahrsschrift der Naturforschenden Gesellschaft in Zürich, Band XXXI. 